\title{
Neutron diffraction, magnetic, and magnetoelectric studies of phase transitions in multiferroic $\mathrm{Mn}_{0.90} \mathrm{Co}_{0.10} \mathrm{WO}_{4}$
}

\author{
I. Urcelay-Olabarria,,${ }^{1,}$ E. Ressouche, ${ }^{2}$ A. A. Mukhin, ${ }^{3}$ V. Yu. Ivanov, ${ }^{3}$ A. M. Balbashov, ${ }^{4}$ G. P. Vorobev, ${ }^{5}$ Yu. F. Popov, \\ A. M. Kadomtseva, ${ }^{5}$ J. L García-Muñoz, ${ }^{6}$ and V. Skumryev ${ }^{7,8}$ \\ ${ }^{1}$ Institut Laue Langevin, 6 rue Jules Horowitz BP 156, F-38042 Grenoble Cedex 9, France \\ ${ }^{2}$ SPSMS, UMR-E CEA/UJF-Grenoble 1, INAC, Grenoble, F-38054, France \\ ${ }^{3}$ Prokhorov General Physics Institute, Russian Academy of Science, Vavilov Street, 38, 119991 Moscow, Russia \\ ${ }^{4}$ Moscow Power Engineering Institute, 105835 Moscow, Russia \\ ${ }^{5}$ M. V. Lomonosov Moscow State University, 119992 Moscow, Russia \\ ${ }^{6}$ Institut de Ciència de Materials de Barcelona, ICMAB-CSIC, Campus Universitari de Bellaterra, E-08193 Bellaterra, Barcelona, Spain \\ ${ }^{7}$ Institució Catalana de Recerca i Estudis Avançats (ICREA), 08010 Barcelona, Spain \\ ${ }^{8}$ Departament de Física, Universitat Autònoma de Barcelona, E-08193 Bellaterra, Barcelona, Spain
}

(Received 16 December 2011; published 30 March 2012)

\begin{abstract}
We have studied various spontaneous and magnetic-field-induced phase transitions in single crystals of multiferroic $\mathrm{Mn}_{0.9} \mathrm{Co}_{0.1} \mathrm{WO}_{4}$ using magnetic and magnetoelectric measurements and neutron diffraction. Compared to pure $\mathrm{MnWO}_{4}$, our data consistently confirm that the anisotropic $\mathrm{Co}^{2+}$ ions induce reorientation of the spin cycloid structure to the $a c$ plane and reveal $P_{a}$ and $P_{c}$ components of spontaneous electric polarization. Field-induced phase transitions accompanied by anomalies of magnetic susceptibility and suppression of both $P_{a}$ and $P_{c}$ polarizations have been observed for $H \| c(\sim 3 \mathrm{~T})$ and $H \| a(\sim 8.5 \mathrm{~T})$. Neutron diffraction has revealed that in both cases the spin cycloid plane flops in direction almost perpendicular to $H$, i.e., close to the $a b$ and $b c$ planes, respectively. Parameters describing the magnetic structures including wave vectors, orientations of the main elliptical axes, etc., have been determined in all spontaneous and field-induced states.
\end{abstract}

DOI: 10.1103/PhysRevB.85.094436

PACS number(s): 75.85.+t, 75.25.-j, 75.50.Ee

\section{INTRODUCTION}

Magnetoelectric multiferroic materials in which magnetic order and ferroelectric order coexist and mutually interact have attracted for a long time much interest because of the possibility to control magnetism by electric fields and vice versa. ${ }^{1-5}$ The recent discovery of a new kind of multiferroic material in which complex magnetic structures induce ferroelectricity has renewed the interest in this field. Magnetic frustration plays an important role in these new materials, since it is at the origin of the observed complex magnetic structures. The coexistence of both magnetic order and electric polarization is tightly linked to symmetry considerations: the magnetic structure breaks the time reversal symmetry, and the appearance of an electric polarization requires a noncollinear spin structure that breaks the spacial inversion symmetry. The mechanisms that drive this phenomena are not completely understood yet; however, in most cycloidal magnetic arrangements, the electric polarization onsets perpendicular to the radial vector $\mathbf{r}_{i j}$ that connects two magnetic moments and to the spin-chirality vector, $\mathbf{S}_{i} \times \mathbf{S}_{j}, \mathbf{S}_{i}$ and $\mathbf{S}_{j}$ being the moments of those atoms: $\mathbf{P} \sim \mathbf{r}_{i j} \times\left(\mathbf{S}_{i} \times \mathbf{S}_{j}\right) .^{6-8}$ The apparent correlation between the polarization and the long-wavelength magnetic structure is often evidenced by magnetic field control on ferroelectric polarization.

$\mathrm{MnWO}_{4}$ belongs to this new class of multiferroic materials that exhibit large magnetoelectric effects. ${ }^{9-11}$ It crystallizes in the wolframite structure (monoclinic space group $P 2 / c$ ). ${ }^{9,10}$ When decreasing temperature, it undergoes three successive phase transitions to different long-range antiferromagnetic states. Below $T_{N}=13.5 \mathrm{~K}$ moments order collinearly along a direction $\hat{u}$ within the $a c$ plane, with a sinusoidal modulation of the amplitudes (so-called AF3 magnetic phase), with $\mathbf{k}=\left(-0.214 \frac{1}{2} 0.457\right)$. The AF2 phase appears in the range $12.5 \mathrm{~K}>T>7.5 \mathrm{~K}$ and presents an additional magnetic component along $b$. The magnetic ordering is thus an elliptical cycloidal spin structure with the same propagation vector $\mathbf{k}$ and with a spontaneous polarization along the $b$ axis. Below $7.5 \mathrm{~K}$ the system is again collinear (along $\hat{u}$ ) and commensurate with $\mathbf{k}=\left( \pm \frac{1}{4} \frac{1}{2} \frac{1}{2}\right)$ (AF1). This succession of magnetic structures is due to a strong competition and subtle balance among multiple magnetic interactions. ${ }^{12}$ Among the three phases, the cycloidal incommensurate AF2 phase between $12 \mathrm{~K}$ and $8 \mathrm{~K}$ is the only one which is also ferroelectric.

Like most multiferroic materials $\mathrm{MnWO}_{4}$ is extremely sensitive to small perturbations induced by external magnetic field or pressure or by chemical substitution. In order to enlarge the temperature range where the multiferroic phase is stable, $\mathrm{MnWO}_{4}$ has been doped with isovalent metal and nonmetal ions. ${ }^{13-20}$ It turned out that doping with $\mathrm{Co}^{2+}$ is particularly interesting since it strongly stabilizes the multiferroic phase at low temperatures. According to preliminary data on powder samples, ${ }^{16}$ the incommensurate multiferroic AF2 phase is expected to substitute the commensurate AF1 ordering at low temperature for cobalt doping above 3\%. At higher doping the $\mathrm{AF} 2$ phase is supposed to transform into the $\mathrm{AF} 2^{\prime}$ magnetic phase having the same propagation vector but with different orientation of the moments. A recent work by Song et al. ${ }^{17}$ on $\mathrm{Mn}_{0.9} \mathrm{Co}_{0.1} \mathrm{WO}_{4}$ single crystals determined that in zero field the magnetic structure of the multiferroic phase consists of a helix that rotates in the $a c$ plane and revealed that the electric polarization lies in the $a c$ plane as well. The phase diagram of further doped compositions is rather complicated: new magnetic phases appear and coexist at the same time, ${ }^{19}$ 
which is a demonstration of the complexity of the cobalt doping in this system.

In this paper we report in detail the evolution of the magnetic structures in zero magnetic field and under magnetic field along the crystallographic axes in $\mathrm{Mn}_{0.9} \mathrm{Co}_{0.1} \mathrm{WO}_{4}$ studied by single crystal neutron diffraction and relate it to the observed magnetic and electric polarization responses to magnetic fields.

\section{EXPERIMENT}

The experiments have been done on single crystals grown by a floating zone method using an FZ apparatus URN-2ZM. ${ }^{21}$ The crystal growth was performed in an air atmosphere at a linear speed of $8-10 \mathrm{~mm} / \mathrm{h}$ with a counter-rotation of crystal and feed rod $\approx 20 \mathrm{~min}^{-1}$. Finally the growing crystal was annealed at $T=1100^{\circ} \mathrm{C}$.

The magnetic response of the crystal to both, ac and dc magnetic fields was studied using the Physical Properties Measuring System and SQUID from Quantum Design. The magnetic field was applied, within few degrees of accuracy, along the principle crystallographic directions of the crystal (shaped as $5 \times 2.5 \times 1 \mathrm{~mm}^{3}$ parallelepiped). No measurable frequency dependence was found in the ac susceptibility in the frequency range of $10 \mathrm{~Hz}$ to $3 \mathrm{kHz}$, which was studied at an ac field amplitude of 10 Oe and at different superimposed dc fields up to $9 \mathrm{~T}$. The data presented here are not corrected for the demagnetizing field effect.

Electric polarization along the principle crystallographic directions was studied by pyroelectric measurements (using an electrometer) in static fields up to $5 \mathrm{~T}$ as well as in pulsed fields up to $20 \mathrm{~T}$. A poling electric field up to $3000 \mathrm{~V} / \mathrm{cm}$ was applied during or prior to the measurement. The dielectric constant was measured by a LCR meter at a frequency of $16 \mathrm{kHz}$. Electrodes were made of conducting silver paste or epoxies with conducting filling. They were deposited on the faces of the studied sample, perpendicular to the direction along which the measurement was performed.

The neutron diffraction experiments were carried out on the two CEA-CRG single crystal diffractometers (D23 and D15) at the Institut Laue Langevin (Grenoble, France), using wavelengths of $\lambda=1.28 \AA$ and $\lambda=1.17 \AA$, respectively. The study in zero magnetic field was done on D23. Complete nuclear and magnetic data collections were made at $17 \mathrm{~K}, 12 \mathrm{~K}$, $9 \mathrm{~K}$, and $2 \mathrm{~K}$ and the temperature dependence of particular peaks was monitored. The experiments in magnetic fields were performed on D15 (with field $H \| c$ ) and D23 (with field $H \| a$ ), and data were collected at $2 \mathrm{~K}$ and $12 \mathrm{~K}$. The absorption in the sample $\left(\mu=0.04 \mathrm{~mm}^{-1}\right)$ has been corrected using the Cambridge Crystallography Subroutine Library ${ }^{22}$ and the structure refinements were done using the FULLPROF SUITE package. ${ }^{23}$ The refinements included an extinction correction, following the model of Becker-Coppens.

\section{ZERO FIELD PHASES}

\section{A. Bulk magnetic response}

The temperature dependence of the dc susceptibility at a field along the principle crystallographic directions is given in Fig. 1(a). A clear maximum, which could be attributed

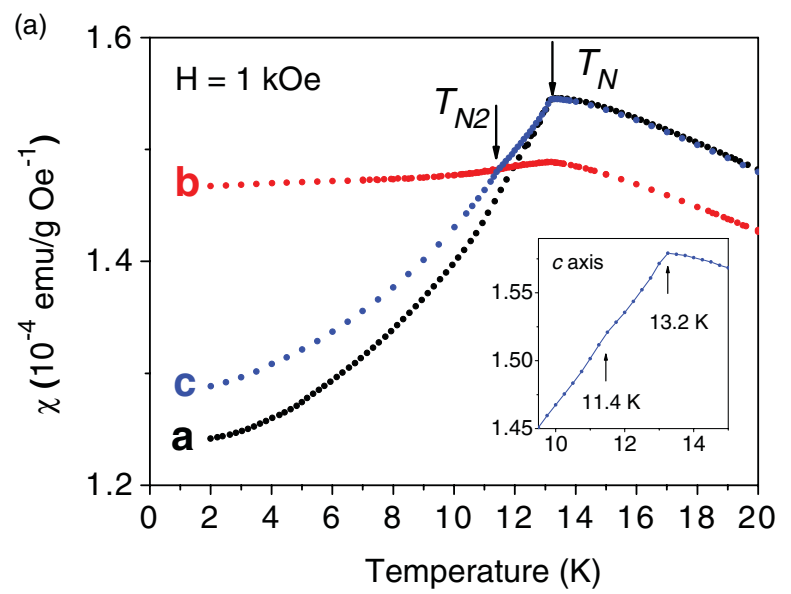

(b)

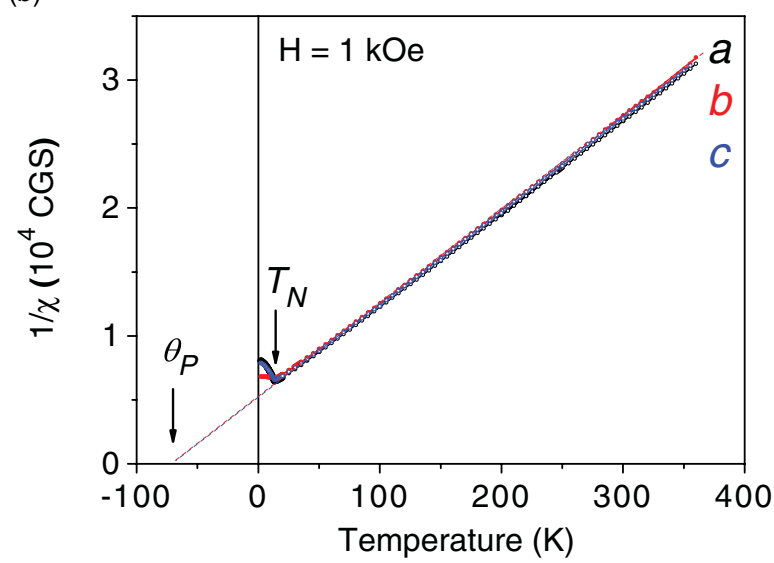

FIG. 1. (Color online) (a) Temperature dependence of dc susceptibility at a field of $1 \mathrm{kOe}$ applied along the principle crystallographic directions. (b) Temperature dependence of the inverse susceptibility. $T_{N}$ and $\theta_{P}$ stand for Neél and asymptotic paramagnetic temperatures, respectively, while $T_{N 2}$ refers to the small change in the susceptibility slope, tentatively ascribed to a second transition.

to the Néel temperature, is seen at $T_{N}=13.2(1) \mathrm{K}$ for all the three directions. It is worth noting that the $a$ - and $c$-axis susceptibilities have a similar value at $T_{N}$, which is bigger than the one for the $b$ axis. With further decrease of temperature another small change in the susceptibility slope takes place at $T_{N 2}=11.4(1) \mathrm{K}$, better visible along the $c$ axis, signaling a second magnetic transition. The two characteristic temperatures are in a good agreement with the data reported in Ref. 17 and associated with appearance of the AF3 phase below $T_{N}$ and with the AF3-AF2' transition at $\mathrm{T}_{N 2}$. Though below $T_{N}$ the $a$-axis susceptibility decreases faster than the one along the $c$ axis, they both change in a similar fashion and in disparity with the relatively small decrease in the $b$-axis susceptibility. The observed finite values of the susceptibility along all crystallographic directions at low temperature differ qualitatively from those of the simple collinear antiferromagnet (where parallel susceptibility vanishes at low temperature) and are somewhat reminiscence of the susceptibility behavior in screw-type magnetic structures, ${ }^{24}$ where both parallel and perpendicular components remain finite.

Above $T_{N}$ the $a$ - and $c$-axis susceptibilities are very close and higher than the $b$-axis susceptibility. The observed 
TABLE I. The crystal structure of $\mathrm{Mn}_{0.90} \mathrm{Co}_{0.10} \mathrm{WO}_{4}$, from refinement of 466 independent reflections collected in the paramagnetic phase $(17 \mathrm{~K})$. The space group is $P 2 / c$ and the atomic positions are the following: Mn and Co at $2 f: \frac{1}{2}, y, \frac{1}{4} ; \mathrm{W}$ at $2 e: 0, y, \frac{1}{4} ;$ and $\mathrm{O}$ at $4 g: x, y, z$. Site occupancies and Debye-Waller factors $\left(\AA^{2}\right)$ are included.

\begin{tabular}{|c|c|c|c|c|c|}
\hline \multicolumn{2}{|c|}{ Cell parameters: } & \multicolumn{2}{|c|}{$\begin{array}{c}a=4.8068(9) \AA, b=5.744(1) \AA \\
c=4.983(5) \AA, \beta=90.91(5)^{\circ}\end{array}$} & \multirow[b]{2}{*}{ Occ. } & \multirow[b]{2}{*}{$B_{e q}$} \\
\hline Atoms & $x$ & $y$ & $z$ & & \\
\hline Mn & & $0.6847(2)$ & & $90.6(2) \%$ & $0.20(3)$ \\
\hline Co & & $0.6847(2)$ & & $9.4(2) \%$ & $0.20(3)$ \\
\hline W & & $0.1802(1)$ & & & $0.16(1)$ \\
\hline $\mathrm{O} 1$ & $0.21142(9)$ & $0.10290(7)$ & $0.9410(4)$ & & $0.29(1)$ \\
\hline $\mathrm{O} 2$ & $0.25150(9)$ & $0.37507(7)$ & $0.3941(4)$ & & $0.29(1)$ \\
\hline \multicolumn{6}{|c|}{ Rel. fac. $\chi^{2}=11.2, R_{F}=2.39, R_{F^{2}}=3.09, R_{F^{2} w}=4.99 \%$} \\
\hline
\end{tabular}

anisotropy is rather different from the one reported in Ref. 17. However, this discrepancy and the magnitude of the measured anisotropy itself should be taken with some precautions until careful measurements on a crystal with similar dimensions are reported or until after proper corrections for sample shape and size are made. ${ }^{25}$ At temperatures above about $30 \mathrm{~K}$ the susceptibility along all the three directions follows Curie-Weiss law [Fig. 1(b)], with a Curie constant per mole of $C=4.15$ (effective magnetic moment $\mu_{\text {eff }}=5.76 \mu_{B}$ ), which is in very good agreement with the expected contribution from the $\mathrm{Mn}^{2+}$ and the effective spin only moment of the $\mathrm{Co}^{2+}$ ions. The paramagnetic Curie temperature $\Theta_{P}=-71(3) \mathrm{K}$ is almost the same for all the crystallographic directions.

\section{B. Neutron diffraction}

$\mathrm{Mn}_{0.9} \mathrm{Co}_{0.1} \mathrm{WO}_{4}$ crystallizes in the $P 2 / c$ (No. 13 , standard setting) monoclinic space group. The cell parameters have been refined by centering 32 nuclear reflections at $17 \mathrm{~K}$ (paramagnetic region) and are the following: $a=4.8068(9) \AA$, $b=5.744(1) \AA, c=4.983(5) \AA$, and $\beta=90.91(5)^{\circ}$. The atomic positions, occupations, and thermal parameters obtained from the refinement of 466 independent reflections collected at $17 \mathrm{~K}$ are summarized in Table I. The difference between the scattering lengths of $\mathrm{Mn}(-3.75 \mathrm{fm})$ and Co $(2.49 \mathrm{fm})$ permitted us the refinement of the occupation factors for these two species, which concluded that there is $9.4(2) \%$ $\mathrm{Co}$ in the sample in good agreement with the expected value of $10 \%$.

The crystal structure is similar to that of pure $\mathrm{MnWO}_{4}$ compound: ${ }^{9}$ it consists of alternating layers of manganese/cobalt and tungsten atoms parallel to the (lllllllane. The oxygen anions form distorted octahedra that are aligned in zig-zag chains along the $c$ axis and the manganese ions are located in the center of the octahedra. The smaller size of $\mathrm{Co}^{2+}$ with respect to $\mathrm{Mn}^{2+}$ ions results in a reduced volume compared to that of the pure $\mathrm{MnWO}_{4}$.

Before describing the magnetic structures, let us expose our notation. In a general picture, an helicoidal magnetic structure is parametrized in the following way. The helix on atom $j$ in unit cell $l$ is described by

$$
\begin{aligned}
\mathbf{m}_{l j}= & \operatorname{Re}_{j}(m) \hat{u}_{j} \cos \left[2 \pi\left(\mathbf{k} \cdot \mathbf{R}_{l}+\varphi_{j}\right)\right] \\
& +\operatorname{Im}_{j}(m) \hat{v}_{j} \sin \left[2 \pi\left(\mathbf{k} \cdot \mathbf{R}_{l}+\varphi_{j}\right)\right],
\end{aligned}
$$

$\mathbf{R}_{l}$ being the vector from the arbitrary origin to the origin of unit cell $l$. The absolute value of each $\varphi_{j}$ is completely arbitrary in the case of an incommensurate propagation vector. However, the difference of phases between the different Bravais sublattices in the unit cell is important. In $\mathrm{MnWO}_{4}$ there are two magnetic atoms in the unit cell, located at $\mathrm{Mn}_{1}$ $\left(\frac{1}{2}, y, \frac{1}{4}\right)$ and at $\mathrm{Mn}_{2}\left(\frac{1}{2}, 1-y, \frac{3}{4}\right), y$ being 0.6853 . In what follows $\Delta \varphi$ stands for the difference $\varphi\left(\mathrm{Mn}_{2}\right)-\varphi\left(\mathrm{Mn}_{1}\right)$. This phase factor is given by representation analysis as developed in Ref. 9 and is equal to $\frac{k_{z}}{2}, k_{z}$ being the component of the propagation vector along $c^{*}$.

$\mathrm{Mn}_{0.9} \mathrm{Co}_{0.1} \mathrm{WO}_{4}$ has two magnetically ordered phases, as revealed by the variation of the integrated intensities of two representative magnetic reflections shown in Fig. 2(a). Below $T_{N} \sim 13 \mathrm{~K}$ it orders in the so-called AF3 phase. Then at $T \sim 11 \mathrm{~K}$, a second transition occurs toward the AF2' phase, as revealed by the accident in the curves of Fig. 2(a) and in agreement with magnetic susceptibility measurements.

After centering 20 magnetic reflections and refining the propagation vector at $12 \mathrm{~K}, 9 \mathrm{~K}$, and $2 \mathrm{~K}$ $\left(\mathbf{k}=\left[-0.222(1) \frac{1}{2} 0.472(1)\right]\right)$, which within error bars is temperature independent, 680 magnetic peaks were collected at each temperature. The magnetic structures were determined by a least-square refinement of the integrated intensities. For the magnetic data treatment the nuclear crystallographic parameters were fixed to the ones in Table I. The scale factor was also fixed to the value obtained by refining the nuclear structure. The details of the obtained magnetic structures are given in Table II. The observed and calculated squared structure factors are plotted in Fig. 3 and the spin arrangements are depicted in Fig. 4. The magnetic structure at $12 \mathrm{~K}$ is sinusoidally modulated (AF3) and the moments lay in the ac plane, along a direction $\hat{u}$ making an angle $\psi=34.7(5)^{\circ}$ $\left(\theta_{u}-90^{\circ}\right)$ with the $a$ axis. They are confined in the equatorial plane of the $\mathrm{MO}_{6}$ octahedra and perpendicular to the $b$ axis [see Fig. 4(a)]. Below $10.8 \mathrm{~K}$ another component $(\hat{v})$ appears perpendicular to the existing one $\hat{u}$. Both $\hat{u}$ and $\hat{v}$, are in the $a c$ plane. As a consequence, the structure becomes an elliptical helix in this $a c$ plane [Figs. 4(b) and 4(c)], the so-called AF2' phase. The appearance of a finite spin chirality is at the origin of the spontaneous polarization observed in our crystal below $T_{N 2}$ (Sec. III C). From the analysis of the magnetic intensities we observe a change in the orientation of the principal axes of the ellipse within the $a c$ plane taking place with decreasing 


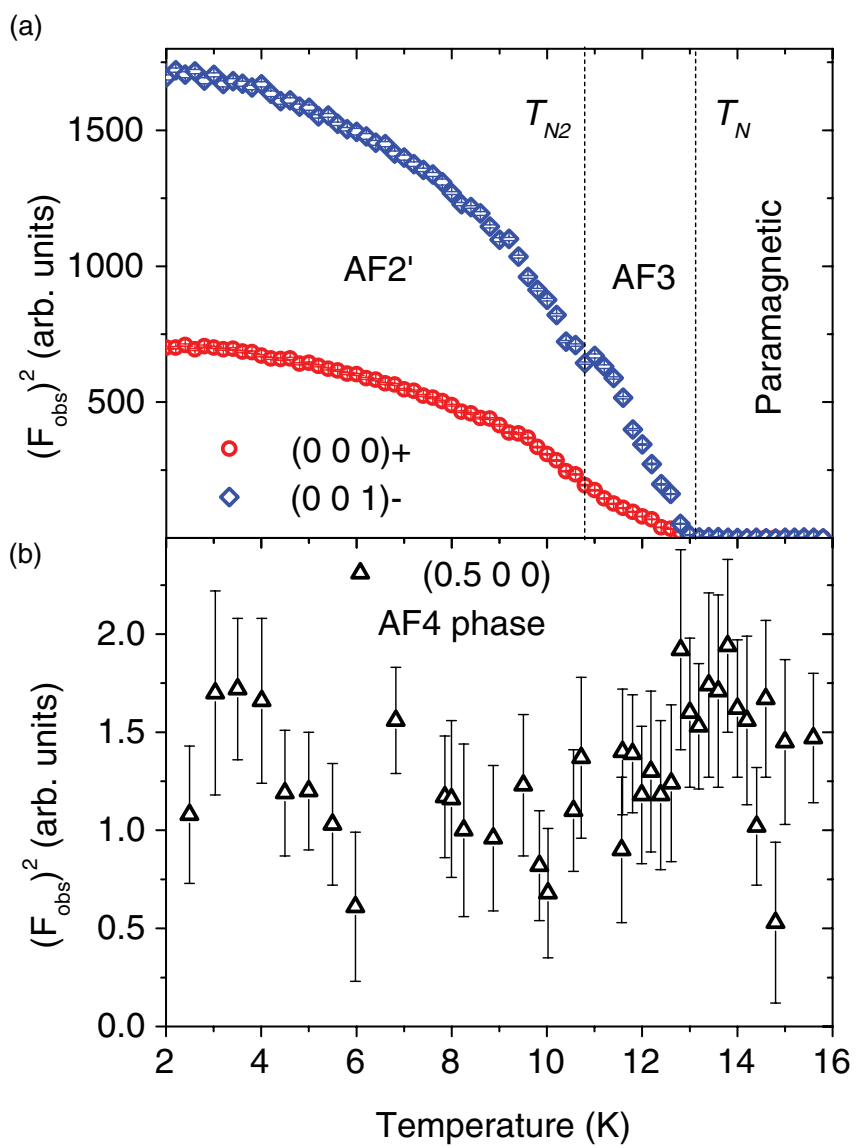

FIG. 2. (Color online) Integrated intensities of magnetic satellites in zero applied field monitored as the temperature increases. (a) Magnetic satellites that corresponds to $\mathbf{k}=[-0.222(1) 0.50 .472(1)]$. The error bars are inside the symbols. Vertical lines indicate the two magnetic transitions. (b) Integrated intensity of $\left(\frac{1}{2} 00\right)$ characteristic of the AF4 commensurate phase.

temperature. The angle that the semimajor axis of the ellipse makes with the $z$ axis decreases from $124.8(5)^{\circ}$ down to $99(2)^{\circ}$ upon cooling from $12 \mathrm{~K}$ to $2 \mathrm{~K}$. Moreover, in the AF2' multiferroic phase there is an apparent evolution of the eccentricity of the ellipse, defined as $\epsilon=\sqrt{1-\frac{\operatorname{Im}(m)^{2}}{\operatorname{Re}(m)^{2}}}$, with temperature. From 9 to $2 \mathrm{~K}$ the eccentricity changes from $\epsilon=0.66$ to 0.42 . The fast development of the magnetic component perpendicular to $\hat{u}$ as the temperature decreases in the $\mathrm{AF} 2^{\prime}$ phase ends up with the creation of a more circular cycloid. We would like to underline the fact that the value obtained in the refinement for $\Delta \phi=0.236(1)$ agrees perfectly with the value expected from representation analysis as developed in Ref. 9, $\frac{k_{z}}{2}$.

The results obtained for our crystal of $\mathrm{Mn}_{0.9} \mathrm{Co}_{0.1} \mathrm{WO}_{4}$ agree with the recent observations of Song et al. ${ }^{16,17}$ on powder samples and single crystals with the same nominal composition. However, there is a discrepancy concerning the values of the magnetic moments' amplitudes. Since our values and the values in Ref. 17 are in a 0.9 ratio, we believe that the difference comes from the site occupation. The value we are giving is an average value of the $\mathrm{Mn}$ site fully occupied. Besides that, we did not observe any
TABLE II. Parameters that describe the magnetic structures of $\mathrm{Mn}_{0.9} \mathrm{Co}_{0.1} \mathrm{WO}_{4}$. Magnetic atoms $\mathrm{Mn}_{1}$ and $\mathrm{Mn}_{2}$ are located in the crystallographic unit cell in $(0.5,0.6846(2), 0.25)$ and $(0.5,0.3154(2)$, $0.75)$, respectively. The unitary vectors $\hat{u}$ and $\hat{v}$ are described by spherical angles: $\theta$ is the angle they make with $z\left(\| c^{*}\right)$ and $\phi$ is the angle that the projection of the unitary vectors in the $x y$ plane make with $x(\| a)$. For a sine wave structure $\operatorname{Im}(m)=0$. The weighted residual factors are $R_{F^{2} w}=13.6 \%, R_{F^{2} w}=6.94 \%$, and $R_{F^{2} w}=6.16 \%$, respectively.

\begin{tabular}{|c|c|c|c|}
\hline & $\begin{array}{c}T=12 \mathrm{~K} \\
(\mathrm{AF} 3)\end{array}$ & $\begin{array}{c}T=9 \mathrm{~K} \\
\left(\mathrm{AF} 2^{\prime}\right)\end{array}$ & $\begin{array}{c}T=2 \mathrm{~K} \\
\left(\mathrm{AF} 2^{\prime}\right)\end{array}$ \\
\hline & \multicolumn{3}{|c|}{$\mathbf{k}=\left[-0.222(1) \frac{1}{2} 0.472(1)\right]$} \\
\hline \multicolumn{4}{|l|}{$\mathrm{Mn}_{1}$} \\
\hline $\operatorname{Re}(m)\left(\mu_{B}\right)$ & $2.533(7)$ & $3.71(1)$ & $4.45(2)$ \\
\hline$\phi_{u}$ & 0 & 0 & 0 \\
\hline$\theta_{u}$ & $124.7(5)^{\circ}$ & $115.5(8)^{\circ}$ & $99(2)^{\circ}$ \\
\hline $\operatorname{Im}(m)\left(\mu_{B}\right)$ & & $2.80(1)$ & $4.03(2)$ \\
\hline$\phi_{v}$ & & 0 & 0 \\
\hline$\theta_{v}$ & & $25.5(8)^{\circ}$ & $9(2)^{\circ}$ \\
\hline $\mathrm{Mn}_{2}$ & & $\begin{array}{l}\mathbf{u}_{2}^{x, z}=-\mathbf{u}_{1}^{x, z} \\
\mathbf{v}_{2}^{x, z}=-\mathbf{v}_{1}^{x, z}\end{array}$ & \\
\hline$\Delta \varphi$ & & $0.236(1)=\frac{k_{z}}{2}$ & \\
\hline
\end{tabular}

presence of the collinear AF4 phase with the propagation vector $\mathbf{k}=\left(\begin{array}{lll}\frac{1}{2} & 0 & 0\end{array}\right)$ known as $\mathrm{AF} 4$, inherent to pure $\mathrm{CoWO}_{4}$. Traces of this phase were reported by Song et al. ${ }^{16}$ for powder $\mathrm{Mn}_{0.9} \mathrm{Co}_{0.1} \mathrm{WO}_{4}$, and it was found to be the main magnetic phase in $\mathrm{Mn}_{0.85} \mathrm{Co}_{0.15} \mathrm{WO}_{4}$ within the interval $9 \mathrm{~K}-16 \mathrm{~K}$ by (a)

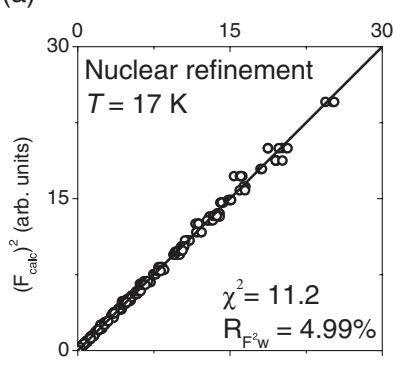

(c)

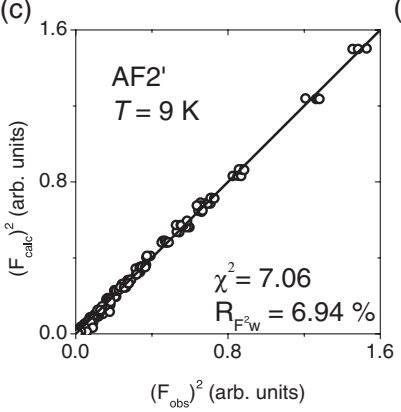

(b)

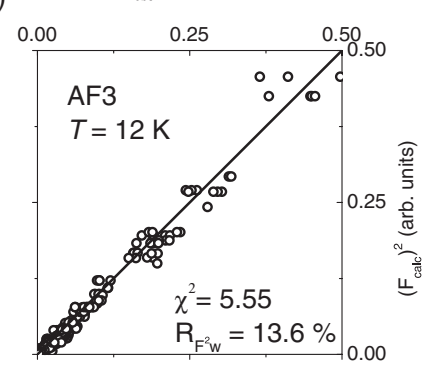

(d)

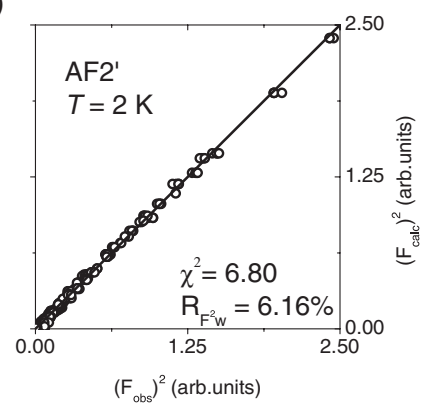

FIG. 3. Agreement plot of the refinements in zero field for (a) the nuclear structure at $17 \mathrm{~K}$, in the paramagnetic phase, (b) the AF3 magnetic phase at $12 \mathrm{~K}$, (c) the AF2' magnetic phase at $9 \mathrm{~K}$, and (d) the AF2' magnetic phase at $2 \mathrm{~K}$. The calculated structure factors are plotted against the experimental ones. The agreement factors $R_{F^{2} w}$ and $\chi^{2}$ are shown. The corresponding magnetic structures are shown in Fig. 4. 
(a) $12 \mathrm{~K}$

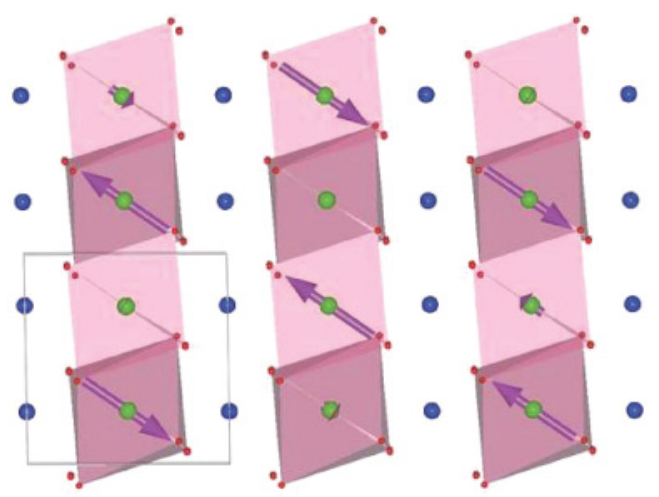

(b) $9 \mathrm{~K}$

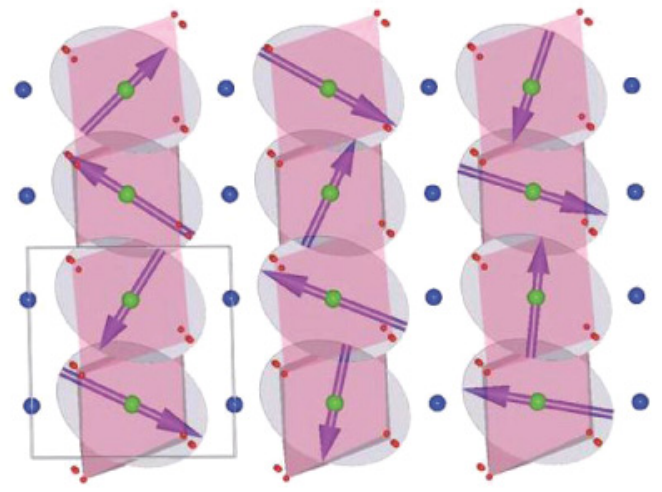

(c) $2 \mathrm{~K}$
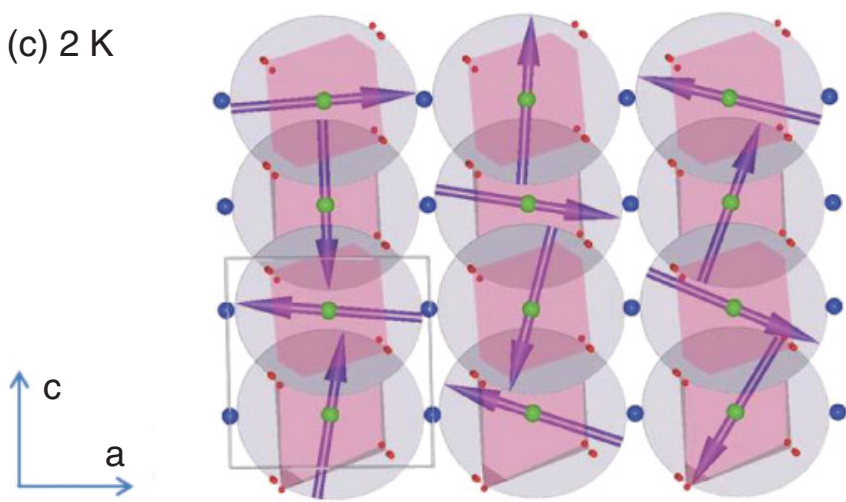

FIG. 4. (Color online) Magnetic structures of $\mathrm{Mn}_{0.9} \mathrm{Co}_{0.1} \mathrm{WO}_{4}$ in zero field, projected in the $a c$ plane. (a) Sinusoidal collinear AF3 magnetic phase at $12 \mathrm{~K}$. AF2' multiferroic phase (b) at $9 \mathrm{~K}$ and (c) at $2 \mathrm{~K}$. The circles in the octahedra (green) correspond to $(\mathrm{Mn}, \mathrm{Co})$ atoms, circles between the octahedra (blue) are $\mathrm{W}$ atoms and the smallest circles (red) are $\mathrm{O}$ atoms. Their relative sizes are not real in the figure for clarity reasons. Distorted $(\mathrm{Mn}, \mathrm{Co}) \mathrm{O}_{6}$ octahedra are shown in purple. The thin gray lines illustrate the crystallographic unit cell. The corresponding magnetic models are described in the text and in Table II.

Chaudhury et al. ${ }^{19}$ Its possible presence in our sample was carefully examined. Figure 2(b) illustrates the absence of the corresponding intensity in $\mathrm{Mn}_{0.9} \mathrm{Co}_{0.1} \mathrm{WO}_{4}$. Our conclusion is that this commensurate phase characterized by $\mathbf{k}=\left(\begin{array}{lll}\frac{1}{2} & 0 & 0\end{array}\right)$ requires higher doping and does not appear in 10\% Co-doped crystal.

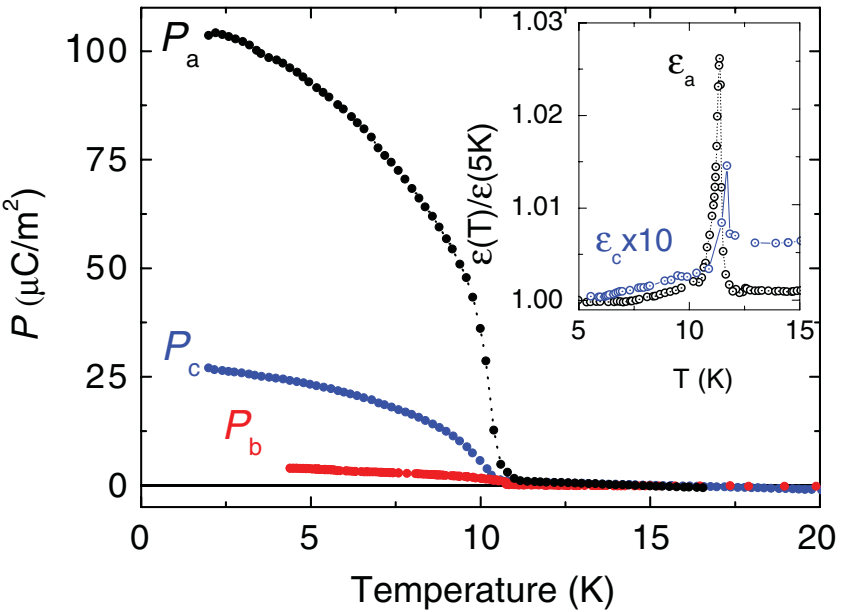

FIG. 5. (Color online) Temperature dependence of spontaneous electric polarization along the principle crystallographic directions which appeared below $T_{N 2}$ in the AF2' cycloidal phase. The insert shows the anomalies of dielectric constants along the $a$ and $c$ axes at $T_{N 2}$ measured at $16 \mathrm{kHz}$.

It should be noticed that all the magnetic structures found at the different temperatures are in perfect agreement with the possible structures predicted by representation analysis as developed in Ref. 9.

\section{Polarization}

The temperature dependence of the electric polarization along $a, b$, and $c$ axes at zero magnetic field is shown in Fig. 5 . Below $T_{N 2} \sim 11 \mathrm{~K}$ electric polarization is observed along both the $a$ and $c$ axes, which increase up to $P_{a} \sim 100 \mu \mathrm{C} / \mathrm{m}^{2}$ and $P_{c} \sim 30 \mu \mathrm{C} / \mathrm{m}^{2}$ at low temperatures. The polarization along the $b$ axis does not exceed $3 \mu \mathrm{C} / \mathrm{m}^{2}$ and is probably due to small misalignment from the $b$ axis. The insert in Fig. 5 shows the temperature variation of the dielectric constant along the $a$ and $c$ axes, which reveals peaks at $T_{N 2}$ where the spontaneous polarization appears; the peak's amplitude along the $a$ axis is significantly higher than that along the $c$ axis. These results unambiguously show that spins in the cycloidal ferroelectric phase $\mathrm{AF2}$ ' are aligned in the $a c$ plane in agreement with our results from neutron diffraction and with Refs. 16 and 17. However we observed polarization not only along the $a$ axis, as expected for the predominated Mn-Mn exchange in zig-zag chains along the $c$ axis, but also along the $P_{c}$ component, the value of which is beyond possible misalignment in the measurement direction and thus suggests a contribution from the exchange along the $a$ axis. This component of the polarization $\left(\sim 20 \mu \mathrm{C} / \mathrm{m}^{2}\right)$ was also observed in Ref. 17. In addition, the spin-wave excitation study on $\mathrm{MnWO}_{4}{ }^{13}$ confirmed that exchange coupling between chains along the $a$ axis is as strong as that along the $c$ axis and similarly strongly frustrated.

\section{FIELD-INDUCED PHASES}

\section{A. Bulk magnetic response}

The field dependence of magnetization at $2 \mathrm{~K}$ along the principle crystallographic directions is shown in Fig. 6. Up 


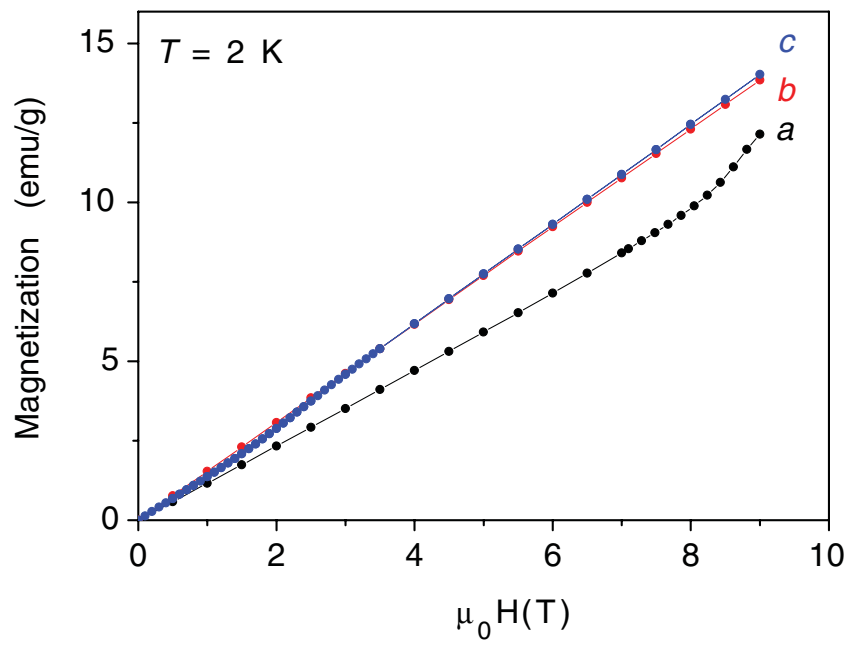

FIG. 6. (Color online) Magnetization curves along the principle crystallographic directions.

to fields as high as $9 \mathrm{~T}$ the magnetization along the $b$ and $c$ axes remains higher than that along the $a$ axis. While the latter changes linearly up to about 7.5 $\mathrm{T}$ it turns concave upward at higher fields signaling a spin reorientation process, presumably of spin-flop type. More careful inspection of the $c$ axis magnetization reveals similar but less pronounced peculiarity at fields of about $2 \mathrm{~T}$. No coercivity was observed in any orientation.

To better monitor the above field-induced departure from the linear variation of magnetization with the field applied along the $a$ and $c$ directions we studied the ac susceptibility at fixed temperatures as a function of the amplitude of the dc field, superimposed in the same direction as the ac field. As illustrated in Fig. 7(a), at $2 \mathrm{~K}$ the field-induced transition along the $a$ direction starts at fields as low as $6.5 \mathrm{~T}$ as evidenced by the increase of the real component of the ac susceptibility, which forms a peak at $8.7 \mathrm{~T}$. This critical field should correspond to the maximum slope in the magnetization curve depicted in Fig. 6. As suggested by Fig. 7(a), the field-induced transition is not completed up to the highest applied field of $9 \mathrm{~T}$. The critical field slightly decreases with increasing temperature (to about $8.3 \mathrm{~T}$ at $10 \mathrm{~K}$ ). A small but noticeable hysteresis is seen depending on the way the field is ramped (see the inset). No clear maximum of ac susceptibility is observed between $T_{N 2}$ and $T_{N}$. However, one should keep in mind that this narrow temperature region is too close to the paramagnetic transition, which might be affected by the relatively high magnetic field. Figure 7(b) focuses on the hardly seen magnetization anomaly along the $c$ direction, depicted in Fig. 6, and demonstrates the versatility of the approach used in studying small changes in the magnetization curve. At $2 \mathrm{~K}$ a clear maximum of the ac susceptibility is observed at about $2.4 \mathrm{~T}$, suggesting a field-induced transition. Such a transition was not reported in the previous studies. ${ }^{17}$ The width of the peak implies that this transition is accomplished in a relatively broad range of fields between about 1 to $3.5 \mathrm{~T}$. A very narrow but noticeable hysteresis is visible in the inset of Fig. 7(b). The peak position is strongly affected by temperature and it shifts to $3.2 \mathrm{~T}$ at $10 \mathrm{~K}$. Importantly the susceptibility peak disappears above $T_{N 2}$.
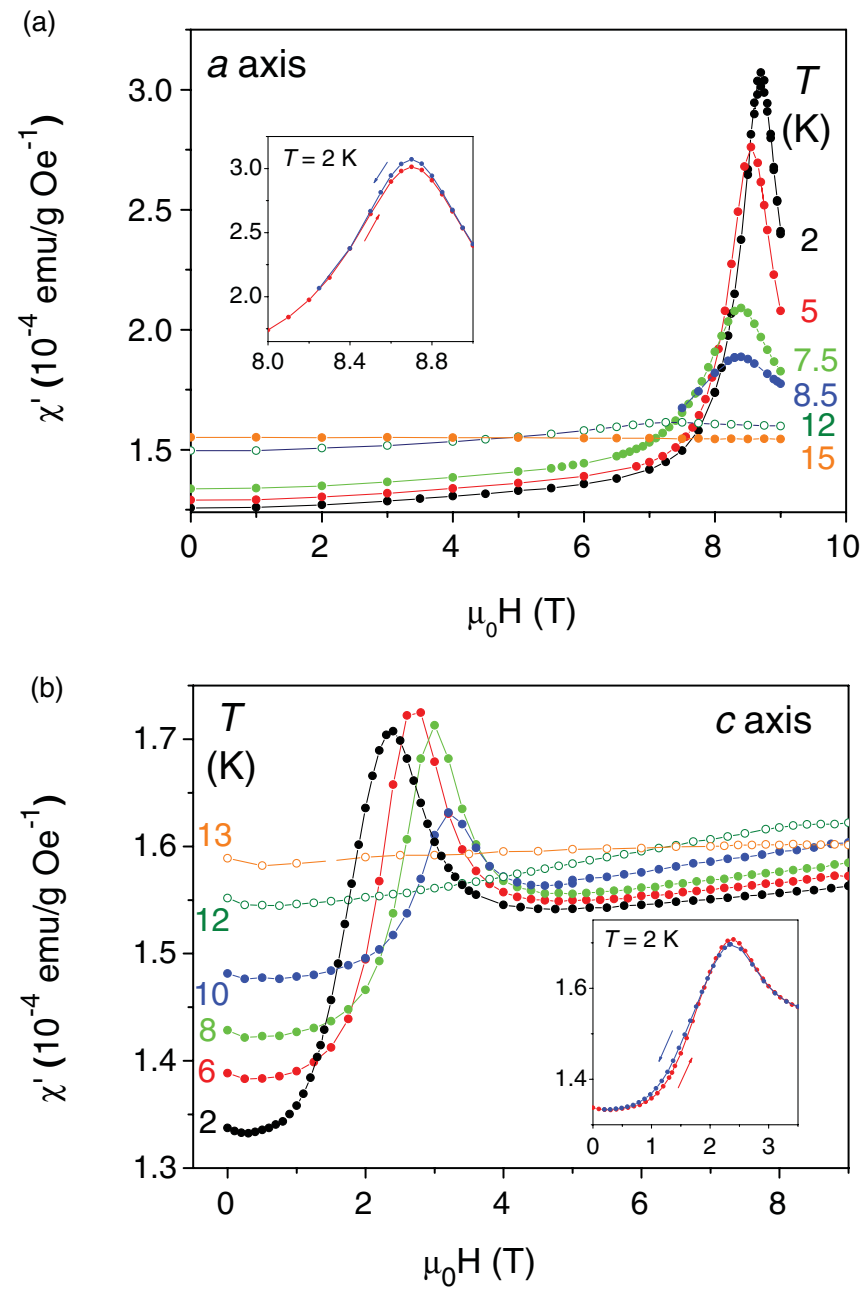

FIG. 7. (Color online) (a) Real component of ac susceptibility along the $a$ axis as a function of superimposed dc field amplitude. (b) The same for the $c$ axis. The insets are zooms of the susceptibility maxima. The arrows indicate the direction of changing the field.

\section{B. Neutron diffraction \\ 1. Field along the $c$ axis}

In an experiment performed on the diffractometer D15, where the sample was mounted with the $c$ axis parallel to the field we have followed the $\left(\begin{array}{lll}0 & 0 & 1\end{array}\right)^{-}$magnetic satellite as a function of the applied field at $T=2 \mathrm{~K}$. The variation of its integrated intensity is depicted in Fig. 8(a). The field-induced transition suggested by the magnetization and ac susceptibility measurements with $H \| c$ is apparent. The transition starts at fields around $\sim 2 \mathrm{~T}$ and is completed at $\sim 3 \mathrm{~T}$, in agreement with those measurements. Data were then collected at 5 T. First, the propagation vector was refined by centering 35 magnetic peaks which revealed that it is nearly unchanged compared to the zero field value: $\mathbf{k}=\left[-0.220(1) \frac{1}{2} 0.469(2)\right]$.

The integrated intensities of 135 independent magnetic peaks were collected at that field and refined. The obtained parameters are gathered in Table III and the corresponding structure is depicted in Fig. 9. Compared to the zero-field structure, there is a flip of the plane where the moments rotate from the $a c$ plane toward the $a b$ plane. The refinements improve if we consider that the rotation plane is not parallel to 


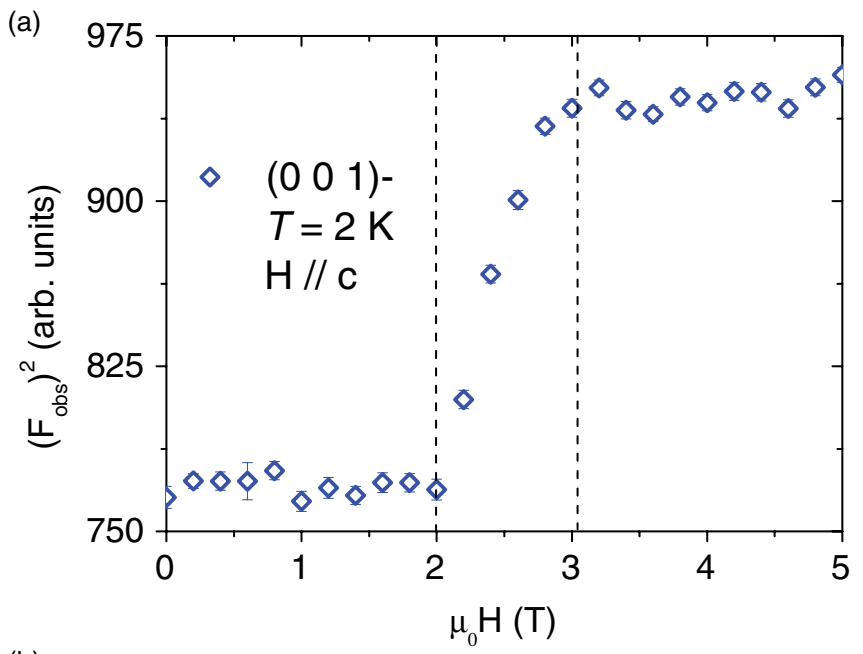

(b)

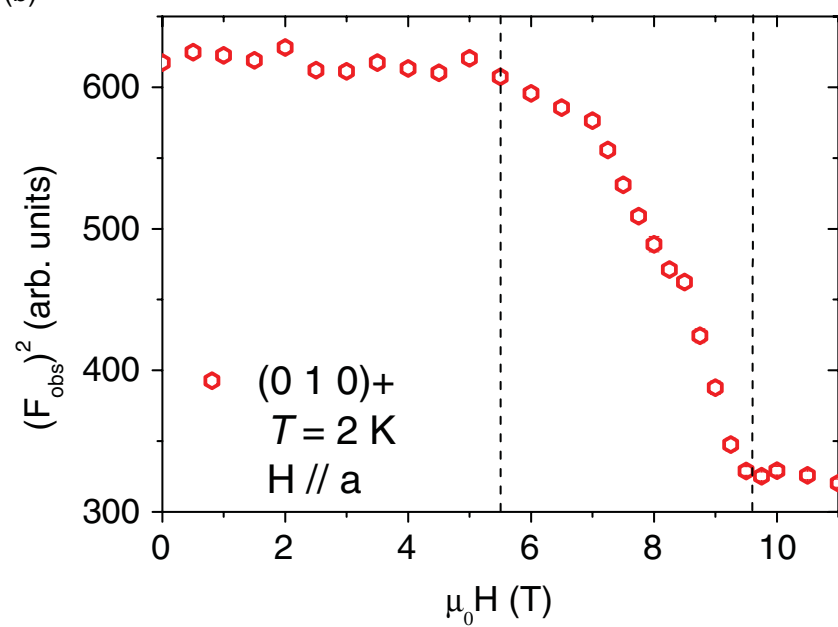

FIG. 8. (Color online) (a) Evolution of the integrated intensity of $\left(\begin{array}{lll}0 & 0 & 1\end{array}\right)^{-}$magnetic satellite with the magnetic field applied parallel to the $c$ axis $(H \| c)$. (b) Similar plot for the $\left(\begin{array}{lll}0 & 1 & 0\end{array}\right)^{+}$satellite when the magnetic field is applied parallel to the $a$ axis $(H \| a)$. The error bars are inside the symbols.

the $a b$ plane but slightly tilted, as the parameters in Table III show. That plane is forced by the field to be as perpendicular as possible to the direction of the external field. In addition, the eccentricity of the ellipse in the new structure has increased, from $\epsilon=0.42(H=0)$ to $0.51(H \| c=5 \mathrm{~T})$.

The influence of the magnetic field on the AF3 phase was also explored. Neutron diffraction data were collected at $12 \mathrm{~K}$ and $5 \mathrm{~T}$. Under these conditions the magnetic structure remains collinear and sinusoidally modulated with the same propagation vector. Only a deviation of the moment direction with respect to the $c^{*}$ axis was observed. The moments remain in the $a c$ plane, but the angle $\theta$ formed with the $c^{*}$ axis decreases: $112(1)^{\circ}$ instead of the $124.8(5)^{\circ}$ at zero field. Hence, the effect of the field along the $c$ axis on AF3 is to rotate the spins toward the $a b$ plane, in order to diminish their projection onto the direction of the external magnetic field. Thus the field moves the spins away from the equatorial plane of the $\mathrm{MO}_{6}$ octahedra. This is in good agreement with the ac susceptibility, from which one should not expect big changes of the AF3 magnetic structure.
TABLE III. Parameters that describe the magnetic structures of the $\mathrm{Mn}_{0.9} \mathrm{Co}_{0.1} \mathrm{WO}_{4}$ at $2 \mathrm{~K}$ under the $5 \mathrm{~T}$ field along the $c$ axis and the $11 \mathrm{~T}$ field along the $a$ axis. The Fourier components of both atoms in the unit cell were considered equal. The weighted residual factors are $R_{F^{2} w}=5.73 \%$ and $R_{F^{2} w}=7.13 \%$, respectively.

\begin{tabular}{|c|c|c|}
\hline $\mathbf{k}$ & 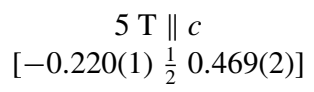 & $\begin{array}{c}11 \mathrm{~T} \| a \\
{\left[-0.206(3) \frac{1}{2} 0.465(1)\right]}\end{array}$ \\
\hline \multicolumn{3}{|l|}{$\mathrm{Mn}_{1}$} \\
\hline $\operatorname{Re}(m)\left(\mu_{B}\right)$ & $4.41(2)$ & $3.67(3)$ \\
\hline$\phi_{u}$ & $-7(2)^{\circ}$ & $90^{\circ}$ \\
\hline$\theta_{u}$ & $95(1)^{\circ}$ & $-21(2)^{\circ}$ \\
\hline $\operatorname{Im}(m)\left(\mu_{B}\right)$ & $3.78(2)$ & $4.19(3)$ \\
\hline$\phi_{v}$ & $83(2)^{\circ}$ & $83(1)^{\circ}$ \\
\hline$\theta_{v}$ & $93(1)^{\circ}$ & $69(2)^{\circ}$ \\
\hline $\mathrm{Mn}_{2}$ & \multicolumn{2}{|c|}{$\begin{array}{l}\mathbf{u}_{2}=-\mathbf{u}_{1} \\
\mathbf{v}_{2}=-\mathbf{v}_{1}\end{array}$} \\
\hline$\Delta \varphi$ & $0.232(2) \sim \frac{k_{z}}{2}$ & $0.237(2) \sim \frac{k_{z}}{2}$ \\
\hline
\end{tabular}

\section{Field along the a axis}

As shown in Figs. 6 and 7, the field applied along the $a$ axis also induces a magnetic transition in the AF2' structure. In this case, a higher field is needed to achieve the spin reorientation and, in addition, the transition is much wider than the one along the $c$ axis. The field dependence of the magnetic satellite $\left(\begin{array}{lll}0 & 1 & 0\end{array}\right)^{+}$, depicted in Fig. 8(b), shows that at $2 \mathrm{~K}$ the transition takes place between 5.5 and $9.5 \mathrm{~T}$. Above $9.5 \mathrm{~T}$ the magnetic transition is completed. We have studied this spin order by collecting neutron data at $11 \mathrm{~T}$ and $2 \mathrm{~K}$ on the D23 diffractometer. Centering of several magnetic reflections concluded that the propagation vector slightly changes compared to the one at zero field becoming $\mathbf{k}=\left[\begin{array}{lll}-0.206(3) & \frac{1}{2} & 0.465(1)\end{array}\right]$. The spins arrangement was determined based on the collected 125 independent reflections. The magnetic structure at $2 \mathrm{~K}$ and $11 \mathrm{~T}$ is shown in Fig. 10 and is described in Table III. The calculated squared structure factors are plotted against the experimental ones in Fig. 11(b). The main effect of the field along $a$ is to flip the plane of the spin cycloid from the $a c$ plane $(H=0)$ toward the $b c$ plane $(H \| a=11 \mathrm{~T})$. Moreover, as seen in Table III, we observe a significant decrease in the amplitude of the ordered moments. In the $H \| a$ configuration, the eccentricity $(\epsilon=0.48)$ is slightly larger compared to the ground state.

The evolution of the AF3 phase with the magnetic field was not studied since from the macroscopic measurements we did not expect any difference but tilting of the moments toward the $b c$ plane, in analogy with field along the $c$ axis.

\section{Polarization}

Let us turn to the electric polarization in the magnetic field. Figure 12(a) shows the dependence of the $a, b$, and $c$ components of the polarization as a function of the magnetic field along the $c$ axis at $T=2 \mathrm{~K}$. A sharp decrease of both $P_{c}$ and $P_{a}$ components is observed beginning at a threshold field of $H_{c} \sim 2 \mathrm{~T}$. With increasing the temperature up to $T_{N 2} \sim 11 \mathrm{~K}$ the evolution of the $P_{c}\left(H_{c}\right)$ and $P_{a}\left(H_{c}\right)$ remains the same with only the initial polarization decreasing and the threshold field increasing. The temperature dependence 
(a)

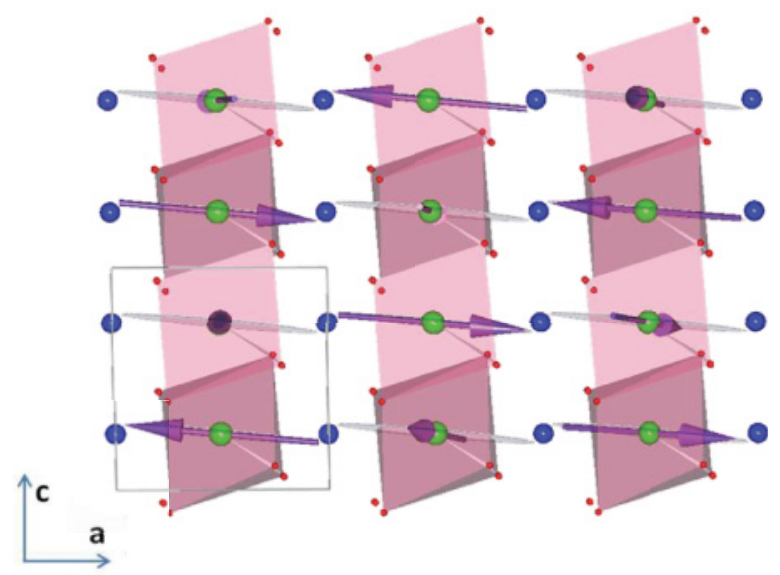

(b)

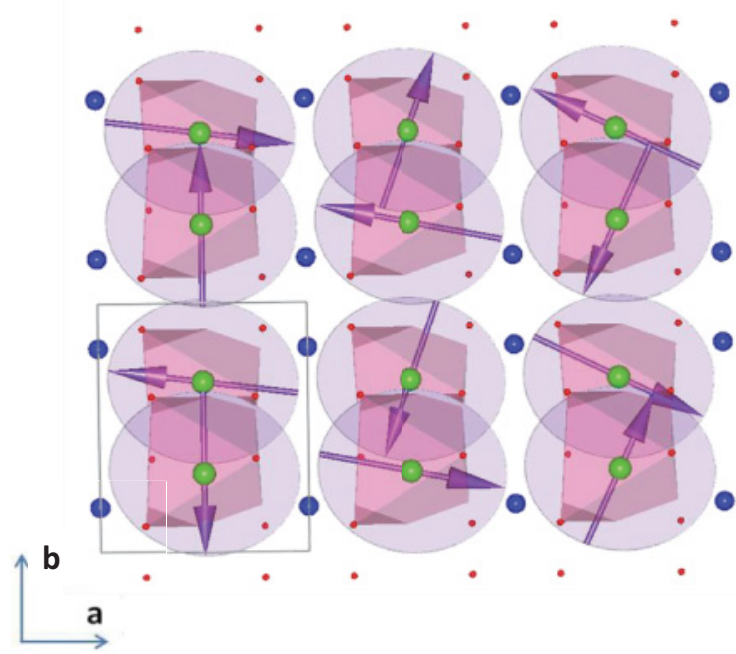

FIG. 9. (Color online) Magnetic structure of $\mathrm{Mn}_{0.9} \mathrm{Co}_{0.1} \mathrm{WO}_{4}$ determined at $2 \mathrm{~K}$ and $5 \mathrm{~T}$ with the magnetic field applied parallel to the $c$ axis $(H \| c)$. The structure is represented by the same color/size code as in Fig. 4. (a) A projection in the $a c$ plane of the spin order. (b) Projection of the magnetic structure in the $a b$ plane. The spin rotation plane is shown in light purple in the magnetic phases induced by the external field. The thin gray frame illustrate the crystallographic unit cell.

of the threshold field for field-induced transition (see the insert in Fig. 12) coincides with the one found from the magnetization and ac susceptibility curves along the $c$ axis (Figs. 6 and 7). The observed disappearance of the $P_{a}$ and $P_{c}$, together with simultaneous increase of the magnetic susceptibility, signals field-induced reorientation of the spin cycloid perpendicular to the field, i.e., to the $a b$ plane. The inverse Dzyaloshiskii-Moriya interaction in the chains along the $a$ axis could contribute to the $P_{b}$ component of the electric polarization in this field-induced phase. However, only a small variation of the $P_{b}$ component was observed at the threshold field which became more noticeable with increasing $T$ (Fig. 12). Whether the small $P_{b}$ polarization is related to the weaker magnetoelectric parameter along the $b$ axis or to a formation of the ferroelectric domains in the field-induced phase (containing both right- and left-handed spin cycloids) remains unclear and requires further study. (a)

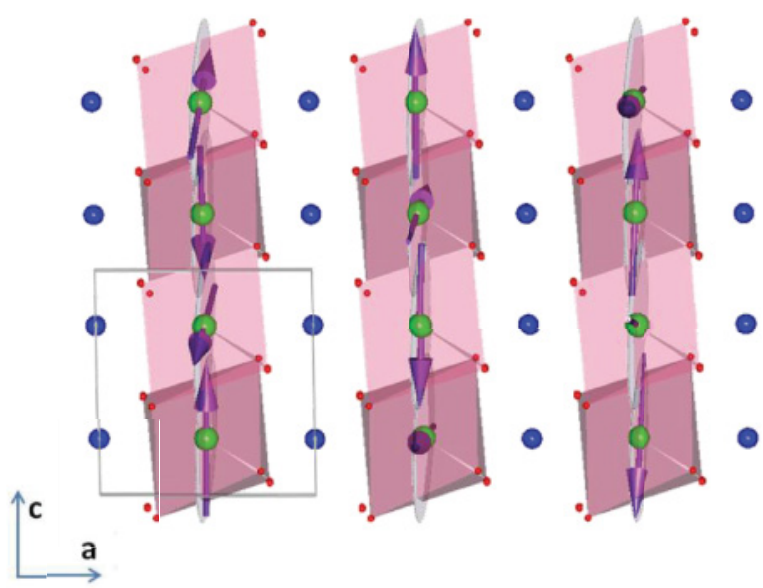

(b)

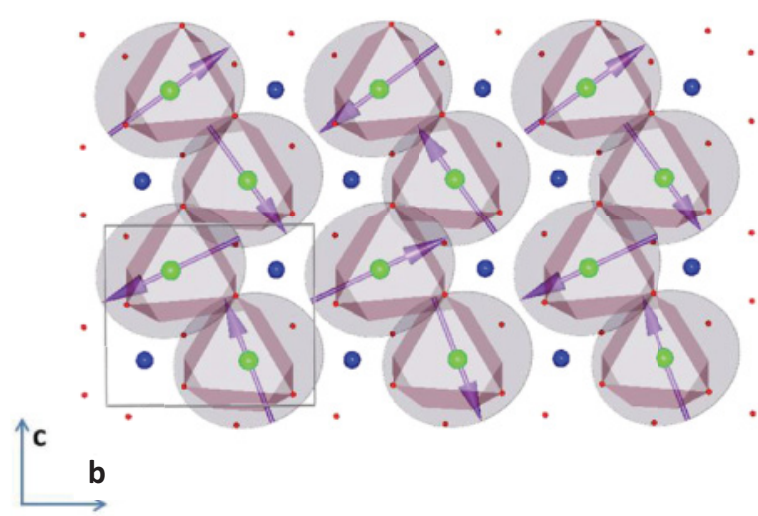

FIG. 10. (Color online) Magnetic structure of $\mathrm{Mn}_{0.9} \mathrm{Co}_{0.1} \mathrm{WO}_{4}$ determined at $2 \mathrm{~K}$ and $11 \mathrm{~T}$ with the magnetic field applied along $a$ $(H \| a)$. Projection of the magnetic structure in the $a c$ plane (a) and in the $b c$ plane (b). The structure is represented by the same color/size code as in Figs. 4 and 9.

When the magnetic field is parallel to the $a$ axis similar suppression of the $P_{a}$ polarization was observed at a pulsed field of $\sim 8.5 \mathrm{~T}$ [Fig. 12(b)]. This is in agreement with the (a)

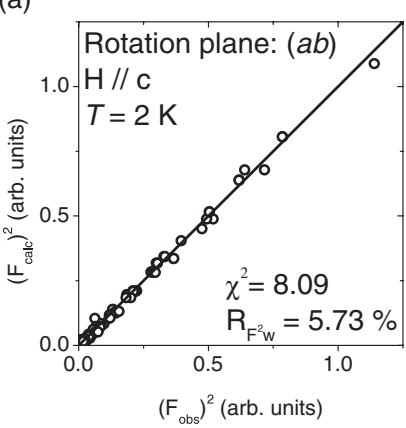

(b)

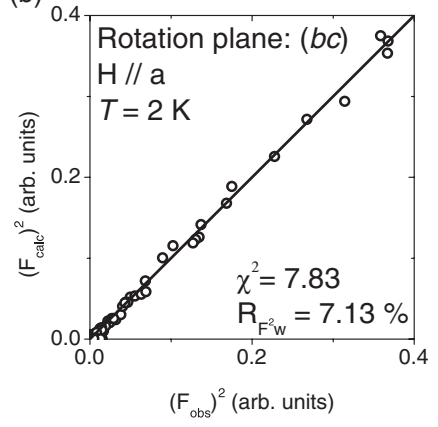

FIG. 11. Agreement plot of the magnetic refinements under the magnetic field in $\mathrm{Mn}_{0.9} \mathrm{Co}_{0.1} \mathrm{WO}_{4}$ at $2 \mathrm{~K}$. (a) Magnetic structure with $H \| c(H=5 \mathrm{~T})$. (b) Magnetic structure with $H \| a(H=11 \mathrm{~T})$. The calculated structure factors are plotted against the experimental ones. The agreement magnetic factors $R_{F^{2} w}$ factor and $\chi^{2}$ are given. The corresponding magnetic models are shown in Figs. 9 and 10. 
(a)

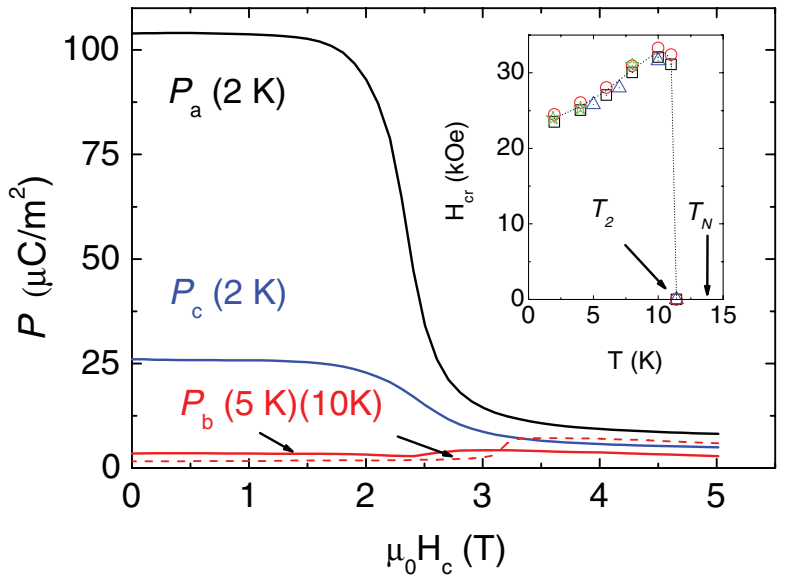

(b)

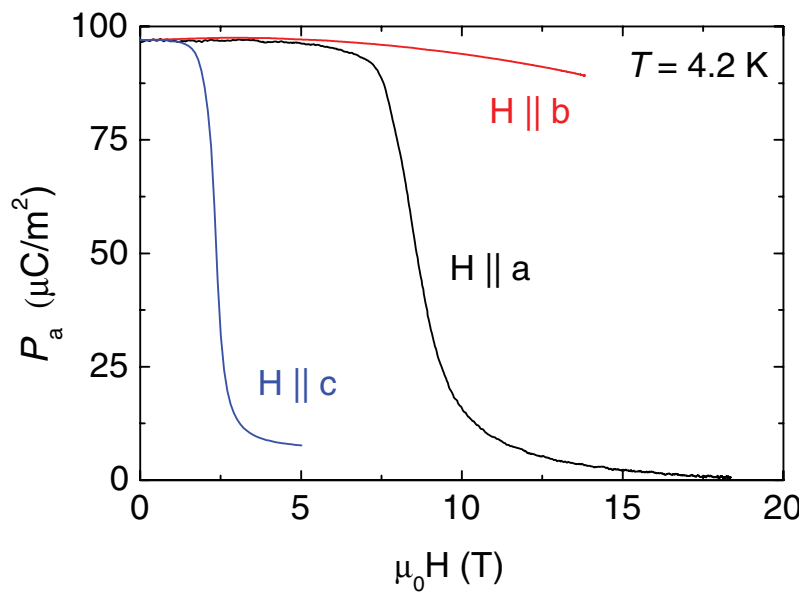

FIG. 12. (Color online) (a) Dependence of the electric polarization along the $a, b$, and $c$ axes under the magnetic field along the $c$ axis. The insert shows the evolution of the critical field as a function of the temperature, $H_{c r}-T$ diagram, obtained from electric polarization and magnetization data. Green stars correspond to magnetic measurements, black squares to $P_{a}$, blue triangles to $P_{b}$, and red circles to $P_{c}$. (b) $P_{a}$ component of the electric polarization in pulsed magnetic field along the $a, b$, and $c$ axes, exhibiting a suppression of $P_{a}$ for $H \| a$ and $H \| c$. Almost nothing changes for $H \| b$.

nonlinear increase of magnetization (Fig. 6) and the maximum in the field dependence of ac susceptibility
[Fig. 7(a)]. It indicates a field-induced spin-flop transition of the cycloid to the $b c$ plane perpendicular to the field.

No substantial effects for the electric polarization was found in the pulsed magnetic field up to $15 \mathrm{~T}$ applied along the $b$ axis confirming the absence of field-induced transitions in this direction, in agreement with the magnetic data in Fig. 6 and with Ref. 16.

\section{SUMMARY AND CONCLUSIONS}

To summarize, the spontaneous and magnetic-fieldinduced phase transitions in single crystals of multiferroic $\mathrm{Mn}_{0.9} \mathrm{Co}_{0.1} \mathrm{WO}_{4}$ were explored using magnetic and magnetoelectric measurements and neutron diffraction. Our data consistently reveal that, at this level of substitution of the $\mathrm{Mn}^{2+}$ ions in the pure $\mathrm{MnWO}_{4}$ by the anisotropic $\mathrm{Co}^{2+}$ ions, the ferroelectric phase with spin cycloid structure in the $a c$ plane and both $P_{a}$ and $P_{c}$ components of spontaneous electric polarization is stabilized up to the lowest temperatures $(\sim 2 \mathrm{~K})$. This phase remains stable in magnetic fields as high as $15 \mathrm{~T}$ applied along the $b$ axis, while in fields along the $c$ and $a$ axes the spin cycloid structure flops from the $a c$ plane to directions almost perpendicular to the magnetic fields at $\sim 3 \mathrm{~T}$ and $\sim 8.5 \mathrm{~T}$, respectively. Those spin reorientations are accompanied by a dramatic suppression of both $P_{a}$ and $P_{c}$ components of the polarization. The refinement of the neutron diffraction data has allowed us to extract characteristic parameters including wave vectors, orientations of the main elliptical axes, etc., of the magnetic structures in the different spontaneous and field-induced phases.

\section{ACKNOWLEDGMENTS}

We are grateful to Virginie Simonet for the critical reading of the manuscript. The authors acknowledge the financial support from MICINN (Spanish government) under Projects No. MAT2009-09308, No. MAT2010-20616-C02-02, and No. CSD2007-00041 (NANOSELECT); Russian Foundation for Basic Researches (Grant No. 09-02-01355, Grant No. 1002-00846, and Grant No. 12-02-01261), and the Ministry of Education and Science of the Russian Federation (Grant No. 16.518.11.7034). We thank the ILL, the French CRG-D23, and the Spanish CRG-D15 projects for the provision of beam time. *urcelay@ill.fr

${ }^{1}$ S. W. Cheong and M. Mostovoy, Nat. Mater. 6, 13 (2007).

${ }^{2}$ T. Kimura, T. Goto, H. Shintani, K. Ishizaka, T. Arima, and Y. Tokura, Nature 426, 55 (2003).

${ }^{3}$ H. Bea, M. Gajek, M. Bibes, and A. Barthelemy, J. Phys.: Condens. Matter 20, 434221 (2008).

${ }^{4}$ S. Ishiwata, Y. Taguchi, H. Murakawa, Y. Onose, and Y. Tokura, Science 319, 1643 (2008).

${ }^{5}$ D. I. Khomskii, J. Magn. Magn. Mater. 306, 1 (2006).

${ }^{6}$ H. Katsura, N. Nagaosa, and A. V. Balatsky, Phys. Rev. Lett. 95, 057205 (2005).
${ }^{7}$ I. A. Sergienko and E. Dagotto, Phys. Rev. B 73, 094434 (2006).

${ }^{8}$ M. Mostovoy, Phys. Rev. Lett. 96, 067601 (2006).

${ }^{9}$ G. Lautenschlager, H. Weitzel, T. Vogt, R. Hock, A. Bohm, M. Bonnet, and H. Fuess, Phys. Rev. B 48, 6087 (1993).

${ }^{10}$ H. Sagayama, K. Taniguchi, N. Abe, T. H. Arima, M. Soda, M. Matsuura, and K. Hirota, Phys. Rev. B 77, 220407(R) (2008).

${ }^{11}$ K. Taniguchi, N. Abe, S. Ohtani, and T. Arima, Phys. Rev. Lett. 102, 147201 (2009).

${ }^{12}$ F. Ye, R. S. Fishman, J. A. Fernandez-Baca, A. A. Podlesnyak, G. Ehlers, H. A. Mook, Y. Wang, B. Lorenz, and C. W. Chu, Phys. Rev. B 83, 140401 (2011). 
${ }^{13}$ E. Garcia-Matres, N. Stüßer, M. Hofmann, and M. Reehuis, Eur. Phys. J. B 32, 35 (2003).

${ }^{14}$ R. P. Chaudhury, B. Lorenz, Y. Q. Wang, Y. Y. Sun, and C. W. Chu, New J. Phys. 11, 033036 (2009).

${ }^{15}$ L. Meddar, M. Josse, P. Deniard, C. La, G. André, F. Damay, V. Petricek, S. Jobic, M.-H. Whangbo, M. Maglione, and C. Payen, Chem. Mater. 21, 5203 (2009).

${ }^{16}$ Y.-S. Song, J.-H. Chung, J. M. S. Park, and Y.-N. Choi, Phys. Rev. B 79, 224415 (2009).

${ }^{17}$ Y.-S. Song, L. Q. Yan, B. Lee, S. H. Chun, K. H. Kim, S. Kim, A. Nogami, T. Katsufuji, J. Schefer, and J.-H. Chung, Phys. Rev. B 82, 214418 (2010).

${ }^{18}$ R. C. Pullar, S. Farrah, and N. McN. Alford, J. Eur. Ceram. Soc. 27, 1059 (2007).
${ }^{19}$ R. P. Chaudhury, F. Ye, J. A. Fernandez-Baca, Y.-Q. Wang, Y. Y. Sun, B. Lorenz, H. A. Mook, and C. W. Chu, Phys. Rev. B 82, 184422 (2010)

${ }^{20}$ R. P. Chaudhury, F. Ye, J. A. Fernandez-Baca, B. Lorenz, Y. Q. Wang, Y. Y. Sun, H. A. Mook, and C. W. Chu, Phys. Rev. B 83, 014401 (2011).

${ }^{21}$ A. M. Balbashov and S. G. Egorov, J. Cryst. Growth, 51, 498 (1981).

${ }^{22}$ J. Brown and J. Matthewman, Cambridge Crystallography Subroutine Library, Report No. RAL93-009, 1993.

${ }^{23}$ J. Rodriguez-Carvajal, Physica B 192, 55 (1993).

${ }^{24}$ Y. Kitano and T. Nagamiya, Prog. Theor. Phys. 31, 1 (1964).

${ }^{25}$ P. Stamenov and J. M. D. Coey, Rev. Sci. Instrum. 77, 015106 (2006). 